\title{
Growth of Metal Nanowhiskers on Patterned Substrate by High Temperature Glancing Angle Deposition
}

\section{AUTHOR(S):}

Suzuki, Motofumi; Hara, Hideki; Kita, Ryo; Hamachi, Kenji; Nagai, Koji; Nakajima, Kaoru; Kimura, Kenji

\section{CITATION:}

Suzuki, Motofumi ...[et al]. Growth of Metal Nanowhiskers on Patterned Substrate by High Temperature Glancing Angle Deposition. Journal of the Electrochemical Society 2009, 157(2): K34-K38

\section{ISSUE DATE:}

2009-12

URL:

http://hdl.handle.net/2433/89642

\section{RIGHT:}

(C) The Electrochemical Society, Inc. 2009. All rights reserved. Except as provided under U.S. copyright law, this work may not be reproduced, resold, distributed, or modified without the express permission of The Electrochemical Society (ECS). The archival version of this work was published in J. Electrochem. Soc., 157(2), K34-K38 (2010).; This is not the published version. Please cite only the published version.; この論文は出版社版でありません。引用の際には出版社版 をご確認ご利用ください。 


\title{
Growth of Metal Nanowhiskers on Patterned Substrate by High-Temperature Glancing Angle Deposition
}

\author{
Motofumi Suzuki, Ryo Kita, Hideki Hara, Kenji Hamachi, Koji Nagai, Kaoru Nakajima, \\ and Kenji Kimura
}

Department of Micro Engineering, Kyoto University, Kyoto 606-8501, JAPAN

In this study, we carry out high temperature glancing angle deposition (HT-GLAD) of Fe and Al on a heated substrate with trench patterns. When vapor is incident perpendicular to the trench direction, nanowhiskers grow only on the surface exposed to the vapor and not inside the trenches. When vapor is incident at a deposition angle larger than $80^{\circ}$ on the sidewall of the trench and not on the substrate surface, nanowhiskers grow only on the sidewall because the condition of deposition at a high temperature and a large deposition angle is satisfied only for the sidewall. Thus, we succeed in the selective growth of nanowhiskers by controlling the geometrical deposition conditions. Further, we also discuss the effect of the local deposition geometry on the growth process. Geometrically selective growth by HT-GLAD is expected to be useful for growing nanowhiskers on nano- and microstructured substrates. 
Unusual Al nanowhiskers grow on the substrate when Al is deposited on a heated substrate at a deposition angle typically larger than $80^{\circ}$, a so-called glancing angle. $(1,2)$ This process is termed high temperature glancing angle deposition (HT-GLAD). In the past, nanowhiskers of various other metals have also been grown by $\operatorname{HT}-\operatorname{GLAD} .(2,3)$ The following conditions must be satisfied for growing nanowhiskers: $(i)$ the deposition temperature must be higher than approximately one-third of the melting point of the metal and (ii) the deposition angle must be typically larger than $80^{\circ}$. Thus, the HT-GLAD growth process is highly sensitive to deposition conditions. Therefore, it would be possible to achieve the selective growth of nanowhiskers on a patterned substrate by this process. There are two approaches to achieve such selective growth as shown in Fig. 1. One approach is based on the shadowing effect (Fig. 1a) and the other is based on the condition that nanowhiskers are grown only at a large deposition angle (Fig. 1b). If vapor is incident obliquely on the patterned substrate, the convex parts of the substrate surface protect its concave parts from the direct impact of incident vapor; therefore, nanowhiskers are expected to grow only on the convex parts, as shown in Fig. 1a. As shown in Fig. 1b, on the other hand, if vapor is incident on certain parts of the substrate (the surface indicated " $\mathrm{A}$ " in Fig. 1b) at a large deposition angle $\theta \geq 80^{\circ}$ and is incident on the other parts (the surface indicated "B" in Fig. 1b) at a small deposition angle $90^{\circ}-\theta$, nanowhiskers grow only on the former parts. However, it is not easy to achieve such geometrically selective growth in conventional nanowhisker growth processes such as chemical vapor deposition based vapor-liquid-solid growth and electrochemical deposition in nanoholes in anodically oxidized aluminum, in which the directivity of the flux of the source materials does not play an important role. Therefore, HT-GLAD is expected to compensate the difficulties to achieve the selective growth by the 
conventional growth processes and to contribute to the development of novel applications of nanowires.

The R\&D of metal nanowires is carried out on a smaller scale than that of the semiconductors and functional oxides. However, metal nanowires have applications in various road maps such as ITRS 2007(4) and potential applications in devices, such as plasmonic waveguides, $(5,6)$ low resistance interconnects, $(4,7)$ nanomechanical memory elements,(8) current-controlled magnetic-domain-wall nanowire logic devices,(9) and field emitters.(10) In many of these applications, the integration of nanowires with threedimensional nano- or microstructures is required. Such integration, i.e., growth of nanowires on nano- or microstructured substrates, is expected to be achieved by carrying out geometrically selective growth using the HT-GLAD technique.

From the viewpoint of fundamental interests, the mechanism of nanowhisker growth by HT-GLAD has not yet been fully understood. Although screw dislocations play an important role in the growth of nanowhiskers, $(11,12)$ there are little experimental observations concerning the atomic process during nanowhisker growth by HT-GLAD. In our previous papers, $(1,2,13)$ we reported that many nanowhiskers grew up to lengths unusually greater than that predicted by a simple growth model proposed by Sears.(11) To understand the growth mechanism of nanowhiskers in detail, it is useful to carry out HT-GLAD on a structured substrate. Oblique deposition results in the formation of illuminated and shadowed regions on the structured substrate so that the transport of the vapor flux near the substrate surface can be modified. An investigation of the growth mode of nanowhiskers by systematically changing geometrical deposition conditions 
would yield an important information on the growth mechanism of nanowhiskers grown by HT-GLAD.

In this paper, we present the selective growth of metal nanowhiskers on a structured substrate achieved by $(i)$ the shadowing effect and (ii) the effect of the incidence angle of vapor flux. In addition, we present results of systematic experiments performed for investigating the geometrical relation between the incident vapor flux and patterns prefabricated on the substrate. Because the growth of nanowhiskers may be related to usual crystal growth mechanisms, their understanding would be useful for understanding the growth mechanisms of nanowhiskers grown by other processes.

\section{EXPERIMENTAL}

Aluminum (purity 99.999\%) or iron (purity 99.99\%) was deposited on a Si substrate with trench patterns in an electron-beam (EB) evaporation apparatus specially designed for carrying out HT-GLAD. After evacuating the preparation chamber to $1 \times 10^{-4} \mathrm{~Pa}$, the substrate, which was placed on a graphite plate, was irradiated (and thus heated) with a halogen lamp. The relation between the substrate temperature measured using thermocouples and that of the halogen lamp was calibrated by preliminary experiments. In the study, by monitoring the temperature of the halogen lamp, the substrate temperature was maintained constant at $390{ }^{\circ} \mathrm{C}$ for $\mathrm{Al}$ or at $470{ }^{\circ} \mathrm{C}$ for $\mathrm{Fe}$ during the deposition process. To reduce the dispersion of the deposition flux, the EB source was placed at a distance of $480 \mathrm{~mm}$ from the center of the substrate. The deposition angle $\alpha$, which is defined as the angle between the substrate normal and the incident direction of 
the deposition flux, was set at an angle of $\alpha \approx 85^{\circ}$ by using an in-vacuum stepper motor.

The thickness $d(\alpha)=d_{0} \cos \alpha$ and deposition rate $\dot{d}(\alpha)=\dot{d}_{0} \cos \alpha$ of Al or Fe were monitored using a quartz crystal monitor, where $d_{0}$ and $\dot{d}_{0}$ are the thickness and deposition rate, respectively, calibrated for films deposited from the normal direction at room temperature. The pressure during the deposition was less than $4 \times 10^{-4} \mathrm{~Pa}$. The detailed deposition geometry, together with the corresponding experimental results, is described in Results and Discussion section. The samples were characterized using a transmission electron microscope (TEM) equipped with an energy dispersive X-ray detector, and a scanning electron microscope (SEM).

\section{RESULTS AND DISCUSSION}

\section{$\underline{\text { Selective Growth of Nanowhiskers by Shadowing Effect }}$}

During HT-GLAD, vapor flux is incident on the substrate at a large deposition angle; therefore, only the convex parts and not the concave parts of the surface are exposed to the vapor flux. Thus, if we use a prepatterned substrate, as is often the case in the selective growth of the columns using low temperature glancing angle deposition, $(14,15)$ we can achieve a patterned growth of nanowhiskers.

In this study, to verify the selective growth of nanowhiskers by the shadowing effect, we carried out the HT-GLAD of $\mathrm{Fe}$ on a $\mathrm{Si}(001)$ substrate with periodic V-shaped grooves along the $<110>$ direction. Fe vapor was incident at a deposition angle of $82^{\circ}$ in such a way that the plane of incidence was perpendicular to the direction of the grooves; 
therefore, most parts of the grooves were shadowed from the Fe vapor. During deposition, the substrate temperature was maintained constant at $470{ }^{\circ} \mathrm{C}$, and the average thickness of deposited Fe was $48 \mathrm{~nm}$.

Figure 2 shows typical SEM images of the resultant samples. As expected, many Fe nanowhiskers with diameters of 50-200 nm and lengths up to $500 \mathrm{~nm}$ grew only on the ridges of the pattern. No significant deposition occured on the shadowed parts of the grooves. This indicates that the diffusion length of the adatoms is considerably shorter than the size of the grooves. For the nucleation of nanowhiskers to occur in HT-GLAD, long-distance diffusion of adatoms is not required. From the SEM images, the diffusion length of adatoms on the surface was estimated to be of the order of $100 \mathrm{~nm}$. Therefore, it would be possible to achieve the selective growth of nanowhiskers by the shadowing effect on nanopatterns with a size down to a few hundred nanometers.

\section{Selective Growth of Nanowhiskers by Control of Deposition Angle}

Because the growth process of nanowhiskers by HT-GLAD is very sensitive to the deposition angle (reported by us in a previous study(1)), in this study, the selective growth of Al nanowhiskers was carried out on sidewalls of rectangular trenches. Aluminum was deposited on a micropatterned $\mathrm{Si}(110)$ substrate with native oxide. Six groups of trenches with nominal widths of $1,3,5,10,15$, and $20 \mu \mathrm{m}$ and a depth of 1.7 $\mu \mathrm{m}$ were prepatterned along the $[1 \overline{1} 0]$ direction. During deposition, the substrate temperature was maintained constant at $390{ }^{\circ} \mathrm{C}$. By selecting appropriate polar and azimuth angles of the substrate during the deposition process, deposition angles on both the surface and the sidewalls of the trenches were controlled independently. Figure $3 \mathrm{a}$ shows schematic representations of the deposition geometry. The polar angle $\alpha$ was 
defined as the angle between the substrate normal and the incident direction of the deposition flux, and the azimuth angle $\phi$ was defined as the angle between the trench direction and the plane of incidence of the Al vapor. As shown in Fig. 3b, the deposition angle on the surface was equal to $\alpha$, whereas that on one of the sidewalls (illuminated sidewall) was $\gamma=\cos ^{-1}(\sin \alpha \sin \phi)$. Samples were prepared at different $\alpha$ values of $55^{\circ}$ and $85^{\circ}$; however, $\gamma$ was fixed at $85^{\circ}$. The average thickness $d$ of Al deposited on the illuminated sidewall was 23 or $30 \mathrm{~nm}$.

Figure 4 shows typical SEM images of a cross section of samples prepared on trenches for [(a) and (b)] $\alpha=55^{\circ}$ and [(d) and (e)] $85^{\circ}$. Thin and long nanowhiskers grow on both types of the samples. Because nanowhiskers grow only under the condition of deposition at a high temperature and at a deposition angle larger than $80^{\circ},(1)$ in $\alpha=55^{\circ}$, they only on the sidewall and not on the surface or the bottom of the trenches. For $\alpha=85^{\circ}$, many nanowhiskers grow on the surface, bottom, and sidewalls of the sample. Thus, the selective growth of nanowhiskers was successfully carried out by controlling the geometrical deposition conditions.

The growth of nanowhiskers on the sidewalls of the trench is not a simple phenomenon. In Fig. 4a and d, it can be observed that nanowhiskers grow not only on the illuminated sidewalls but also on the shadowed sidewalls that have never been directly exposed to the vapor flux. The growth of nanowhiskers on the shadowed sidewalls is not exceptional, while the number of nanowhiskers grown on the shadowed part of the bottom is small. Figure 5 shows the SEM images captured from various angles for the same two samples - : with $\alpha=55^{\circ}$ (Fig. 5a) and $\alpha=85^{\circ}$ (Fig. 5b-d). As observed in Fig. 
$5 \mathrm{a}$ and $\mathrm{b}$, the nanowhiskers grow on the shadowed sidewalls of both the samples. Further, many islands or grains as well as nanowhiskers are present on the surface, part of the bottom exposed to the incident vapor, and on illuminated sidewalls. The morphological features observed in Fig. 5c are characteristic of the samples prepared by HT-GLAD, as reported in our previous papers. $(1,2)$ However, no recognizable islands or grains are present on the shadowed sidewalls, and only thin and long nanowhiskers grow on these sidewalls (Fig. 5d). Few nanowhiskers grow on the shadowed part of the bottom (Fig. 5b and d). This suggests that the re-evaporation or reflective scattering of atoms deposited on the sidewalls of the trenches contributes to the growth of nanowhiskers.

\section{Effects of Local Deposition Geometry on Growth of Nanowhiskers}

In addition to the growth of nanowhiskers on the shadowed sidewalls, the size and number of nanowhiskers growing on the sidewalls depend strongly on $\alpha$. Figure 6 shows a plan view of samples prepared at $\alpha=73^{\circ}, 84^{\circ}$, and $87^{\circ}$. As schematically indicated under each SEM image, the incident direction of Al vapor under the condition of large $\alpha$ is more parallel to the trenches than that under the condition of small $\alpha$. At $\alpha=73^{\circ}$, only a few short nanowhiskers grow on the sidewalls, whereas at large $\alpha$, many long nanowhiskers grow on the sidewalls of the trenches. Figure 7 shows the relation between $\alpha$ and the number density of nanowhiskers grown on the sidewalls for the samples with an average Al thickness of $30 \mathrm{~nm}$. Clearly, the number of nanowhiskers drastically increases for $\alpha \geq 80^{\circ}$.

Next, we discuss the effect of the deposition geometry on the growth of Al nanowhiskers. Because $\gamma$ is fixed to $85^{\circ}$ and the amount of $\mathrm{Al}$ deposited on the sidewall is constant at $30 \mathrm{~nm}$, the number and size of the nanowhiskers should not change if 
deposition is carried out on a large flat substrate. Ordinary diffusion processes cannot satisfactorily explain this $\alpha$ dependence of the number of nanowhiskers. In the deposition on the sidewalls of the trenches, the distance from the trench edge to the growth point changes significantly with a change in $\alpha$. If atoms deposited in front of the growing nanowhiskers contribute to the growth by re-evaporation or scattering, the dependence of the number and size of nanowhiskers on $\alpha$ may be understood.

In fact, it has been reported that the sticking coefficient of metals on some oxide substrates is extremely small even at a few hundred degrees Celsius. For example, Benck et al.(16) reported the sticking of $\mathrm{Ag}$ deposited on some oxides as a function of the substrate temperature. The significant amount of $\mathrm{Ag}$ was deposited at large deposition angles in their experimental set up. Remarkably, even at $300{ }^{\circ} \mathrm{C}$, the sticking coefficient of $\mathrm{Ag}$ on $\mathrm{SiO}_{2}$ is smaller than 0.3. The sticking coefficient of $\mathrm{Al}$ on $\mathrm{SiO}_{2}$ may be similar. If atoms reflected on the surface in front of the growing nanowhiskers also contribute to their growth, the growth rate increases significantly.

We used a conventional growth model $(1,11,17)$ to estimate the contribution of reflected atoms to the nanowhisker growth. Figure 8 shows the growth model of nanowhiskers on the sidewall of a trench. In this conventional growth model, adatoms diffuse over the sides of the nanowhiskers and are incorporated at the top of the nanowhiskers. As an extreme case of the fastest growth, we assume that all atoms impinging upon the nanowhiskers are incorporated into the nanowhiskers and that their radius does not change during the growth process. Evaporated atoms are directly incident on the side of a nanowhisker of length $l$ growing at a distance $w$ from the edge of the substrate. Here, the vapor flux incident on the surface of the side of the nanowhisker is 
$f \tan \gamma$, where $f$ is the component of the vapor flux perpendicular to the surface. We assume that the atoms reflected on the surface in front of the nanowhisker also contribute to the nanowhisker growth. Here, we also assume that the reflectance of the atoms is $s$. The length of the nanowhisker depending on the reflectivity of the atoms is written as

$$
l= \begin{cases}l_{0} \exp \left\{\frac{2(1+s) \tan \gamma}{\pi r} d\right\}, & (l \tan \gamma<w) \\ (1+s) l_{c} \exp \left\{\frac{2 \tan \gamma}{\pi r}\left(d-d_{c}\right)\right\}-s l_{c}, & (l \tan \gamma \geq w)\end{cases}
$$

where $l_{0}$ is the initial length of the nanowhiskers, namely, the height of the nuclei, and $r$ is the radius of the nanowhiskers. At $d=d_{c}, l=l_{c}=w / \tan \gamma$. For nanowhiskers growing on the sidewall at a depth $w_{0}$ from the surface, $w=w_{0} \sin \gamma / \cos \alpha$. We used this equation to calculate the nanowhisker length as a function of $\alpha$ for some values of $s$, as shown in Fig. 9. In these calculations, we assumed very small nuclei with $l_{0}=0.1 \mathrm{~nm}$. In addition to assuming the height of nuclei, we assumed typically used values or typically observed values in the experiments $\left(d=30 \mathrm{~nm}, r=30 \mathrm{~nm}, w_{0}=50 \mathrm{~nm}\right.$, and $\left.\gamma=85^{\circ}\right)$. For $s=0$, i.e., in the case that the reflected atoms do not contribute to nanowhisker growth, the length of the nanowhiskers is independent of $\alpha$. The length of nanowhiskers grown at $s=0$ is small. For $s \neq 0$, i.e., in the case that the reflected atoms contribute to the nanowhisker growth, long nanowhiskers can grow from a small nucleus. Further, in this case, the length of the nanowhiskers depends on $\alpha$. Under the condition where the long nanowhiskers grow, it may be easy to find many nanowhiskers. This result is consistent with the experimental results shown in Fig. 7. 
The above-mentioned model is also useful in understanding the growth mechanism of very long nanowhiskers on substrates with rough surfaces.(13) In our previous paper, we reported that there are a significant number of nanowhiskers with lengths considerably greater than that predicted by the conventional model ( $s=0$ in Eq. 1$)$ and that the number of long nanowhiskers is independent of the surface roughness under the same deposition condition with this study except for the substrate.(13) In fact, if $s=0$, from Eq. 1, the length $l_{0}$ of the nucleus should be approximately $250 \mathrm{~nm}$ for the growth of the typical long nanowhisker reported in Ref. (13) having $l=7 \mu \mathrm{m}$ and $2 r=100 \mathrm{~nm}$ at $d=23 \mathrm{~nm}$ and $\gamma=85^{\circ}$. In practice, not all adatoms might be incorporated into the nanowhiskers, and the diameter of the nanowhiskers could increase; therefore, the growth rate of nanowhiskers should be smaller than that predicted by Eq. 1. Clearly, results yielded by the conventional model are inconsistent with the observation of the growth of long nanowhiskers in our study. It is strongly suggested that a significant amount of Al is supplied to the nanowhiskers both via direct deposition on their side surface and from the surrounding region. However, ordinary surface diffusion cannot explain the fact that long nanowhiskers can grow on a very rough surface. If we take into account the contribution of atoms reflected on the surface in front of nanowhiskers to nanowhisker growth, long nanowhiskers can easily grow from small nuclei. The growth of nanowhiskers on the shadowed sidewall may be attributed to the contribution of reflected or scattered atoms. Therefore, we can say that atoms reflected or scattered on the surface in front of nanowhiskers significantly contribute to the growth of long nanowhiskers by HT-GLAD.

\section{CONCLUSION}


We carried out the HT-GLAD of Fe and Al on a heated substrate with trench patterns. Nanowhiskers selectively grew on the substrate by the shadowing effect and under controlled geometrical deposition conditions. In growth of $\mathrm{Al}$ nanowhiskers on the sidewalls of the trenches, nanowhiskers grew not only on illuminated sidewalls but also on shadowed sidewalls. The size and number of nanowhiskers growing on the sidewalls depended strongly on $\alpha$, although $\gamma$ was maintained constant. The reflective scattering of atoms on the surface in front of nanowhiskers plays an important role in the growth of nanowhiskers.

\section{Acknowledgments}

We are grateful to Sadamu Kinoshita for the SEM observations. We are thankful to Akio Takada of SONY Corporation for providing the patterned substrates. 


\section{References}

1. M. Suzuki, K. Nagai, S. Kinoshita, K. Nakajima, K. Kimura, T. Okano and K. Sasakawa, Appl. Phys. Lett., 89, 133103 (2006).

2. M. Suzuki, K. Nagai, S. Kinoshita, K. Nakajima, K. Kimura, T. Okano and K. Sasakawa, J. Vac. Sci. Technol. A, 25, 1098 (2007).

3. M. Suzuki, K. Hamachi, K. Nagai, R. Kita, K. Nakajima and K. Kimura, Mater. Res. Soc. Symp. Proc., 1058E, 1058 (2008).

4. The International Technology Roadmap for Semiconductors, ITRS 2007 EDITION (2008).

5. A. Manjavacas and F. J. Garcia de Abajo, Nano Letters, 9, 1285 (2009).

6. S.-D. Liu, M.-T. Cheng, Z.-J. Yang and Q.-Q. Wang, Opt. Lett., 33, 851 (2008).

7. L. J. Chou, Y. L. Chueh and M. T. Ko, Thin Solid Films, 515, 8109 (2007).

8. R. L. Badzey, G. Zolfagharkhani, A. Gaidarzhy and P. Mohanty, Appl. Phys. Lett., 85, 3587 (2004).

9. $\quad$ S. S. P. Parkin, United States Patent No. 6,834,005 (Dec. 21 2004).

10. Y. L. Chueh, L. J. Chou, S. L. Cheng, L. J. Chen, C. J. Tsai, C. M. Hsu and S. C. Kung, Appl. Phys. Lett., 87, 223113 (2005).

11. G. W. Sears, Acta Met., 3, 361 (1955).

12. M. J. Bierman, Y. K. A. Lau, A. V. Kvit, A. L. Schmitt and S. Jin, Science, 320, 1060 (2008).

13. K. Hamachi, M. Suzuki, K. Nakajima and K. Kimura, Mater. Res. Soc. Symp. Proc. , 1059E, 1059 (2008).

14. B. Dick, M. J. Brett, T. Smy, M. Belov and M. R. Freeman, J. Vac. Sci. Technol. $B, 19,1813$ (2001).

15. T. M. Lu, D. X. Ye, T. Karabacak and G. C. Wang, Mater. Res. Soc. Symp. Proc., 849, 13 (2005).

16. R. F. Benck, C. Crisco and H. L. Spies, J. Vac. Sci. Technol., 10, 562 (1973).

17. A. J. Melmed and R. Gomer, J. Chem. Phys., 34, 1802 (1961). 


\section{FIGURES}

Figure 1. Approaches of the selective growth of nanowhiskers based on (a) shadowing and (b) deposition-angle dependence of the growth of nanowhiskers.

Figure 2. SEM images of Fe deposited on V-shaped trenches: (a) Bird's-eye view and (b) cross-sectional view. The arrow indicates the incident direction of Fe vapor.

Figure 3. Schematic drawings of deposition geometry. (a) Relation between the vapor source and the substrate and (b) incident direction of the vapor flux relative to the trench structure.

Figure 4. SEM images of cross sections of [(a) and (b)] the sample with $\alpha=55^{\circ}$ and $\gamma=85^{\circ}$ and $\left[(\mathrm{d})\right.$ and (e)] the sample with $\alpha=85^{\circ}$ and $\gamma=85^{\circ}$. The deposition geometries are also shown schematically in (c) and (f). For both samples, the average thickness of deposited Al was $23 \mathrm{~nm}$.

Figure 5. SEM images of the sample with $\alpha=55^{\circ}$ and $\gamma=85^{\circ}$ [(a) plan view] and the sample with $\alpha=85^{\circ}$ and $\gamma=85^{\circ}$ [(b) plan view, (c) illuminated sidewall and bottom, and (d) shadowed sidewall and bottom]. In these images, characteristic parts such as surface, bottom, illuminated sidewall, and shadowed sidewall are marked as "Su," "B," "I," and "Sh," respectively.

Figure 6. SEM images of the plan view of samples with (a) $\alpha=73^{\circ}$, (b) $\alpha=84^{\circ}$, and (c) $\alpha=87^{\circ}$. The average thickness of deposited Al is $30 \mathrm{~nm}$; further, $\gamma=85^{\circ}$ for all samples. The deposition geometries are indicated schematically under each SEM image. 
Figure 7. Relation between $\alpha$ and the number density of nanowhiskers growing on the sidewalls for samples with average Al thickness of $30 \mathrm{~nm}$.

Figure 8. Growth model of nanowhiskers on the sidewall of the trench.

Figure 9. Calculated results of the nanowhisker length as a function of $\alpha$ for various values of $s$. In these calculations, we assumed $l_{0}=0.1, d=30, r=30, w_{0}=50 \mathrm{~nm}$, and $\gamma=85^{\circ}$ 


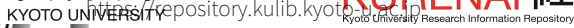

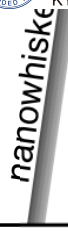

vapor

\section{patterned substrate}

(b)

\section{shadow}

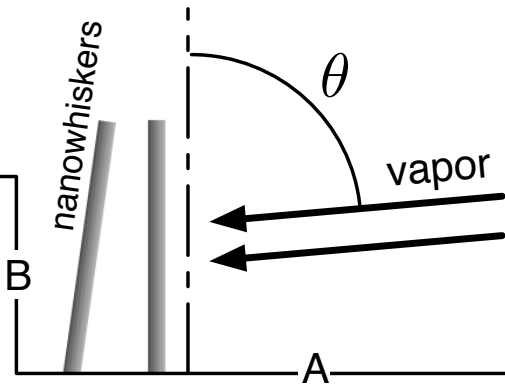

patterned substrate 


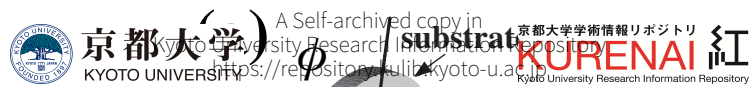

\section{trenches}

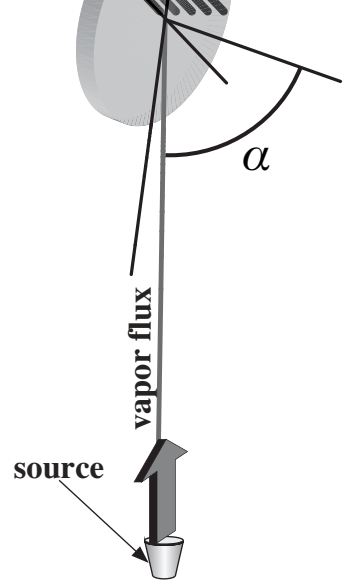

(b)
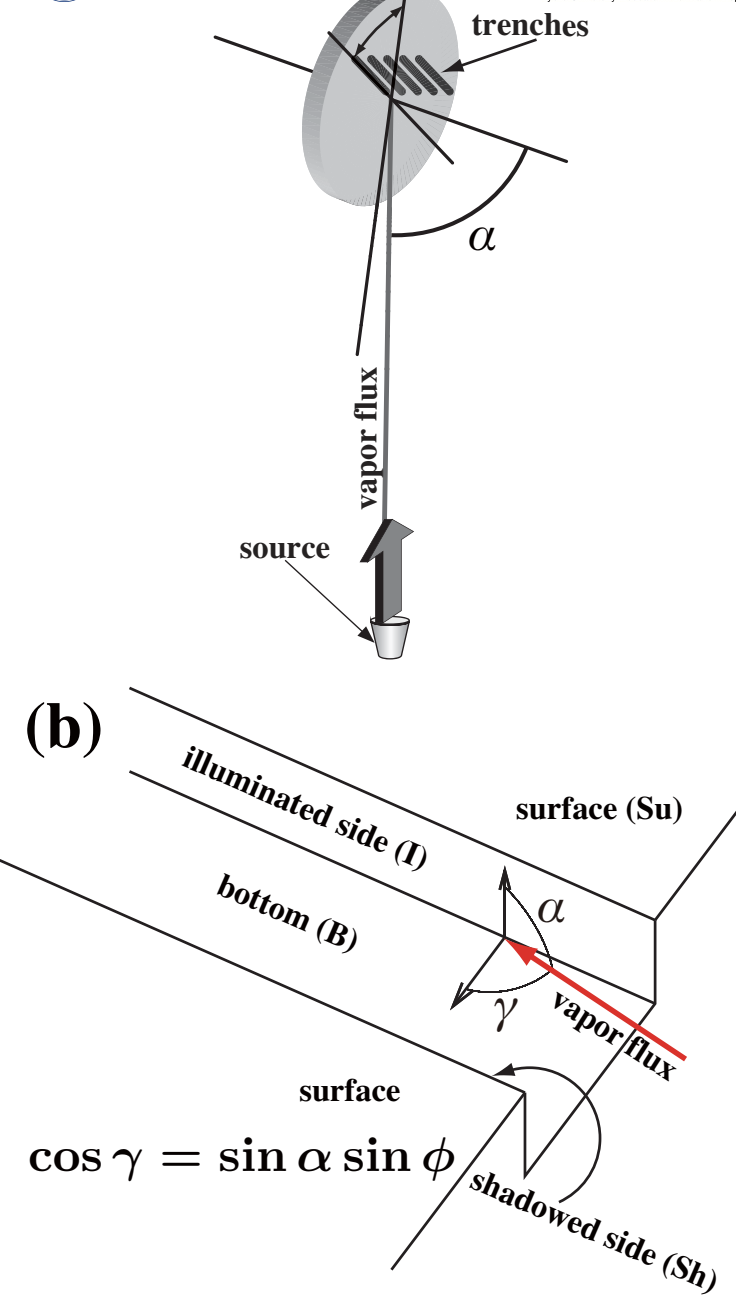

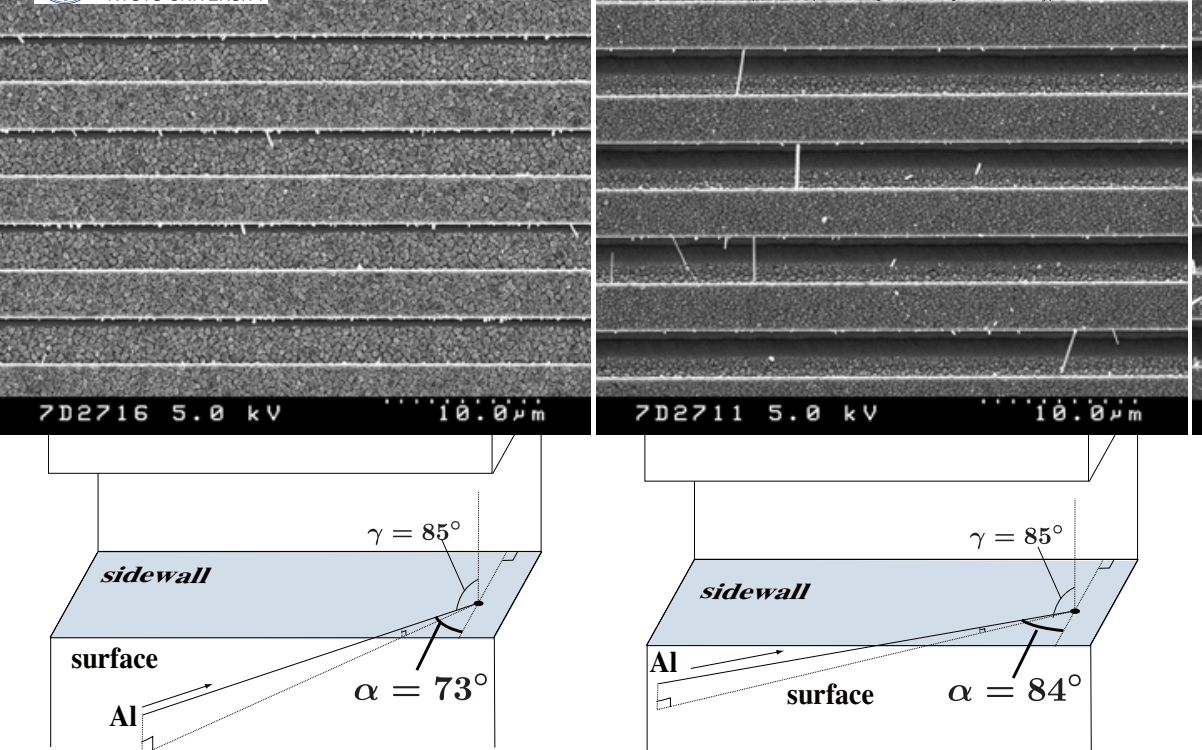

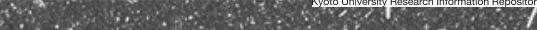

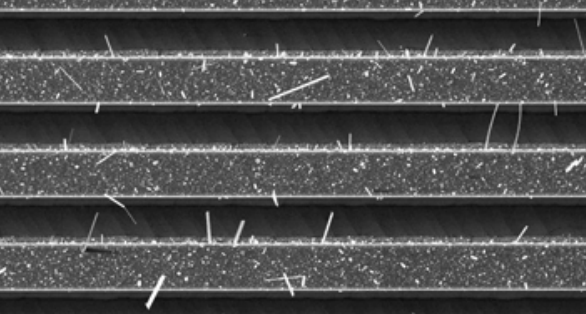

$7 \mathrm{D} 27115.0 \mathrm{kV}$

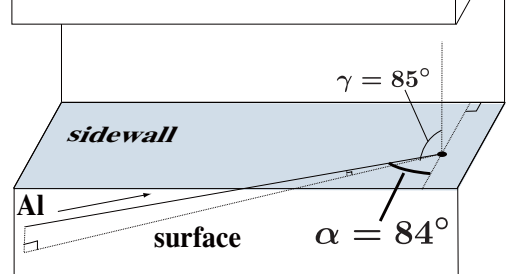

$7 N 19165.0 \mathrm{kV}$

$\gamma=85^{\circ}$

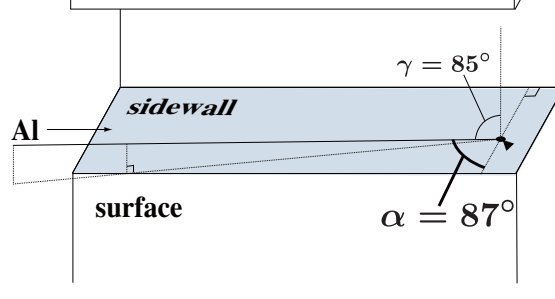




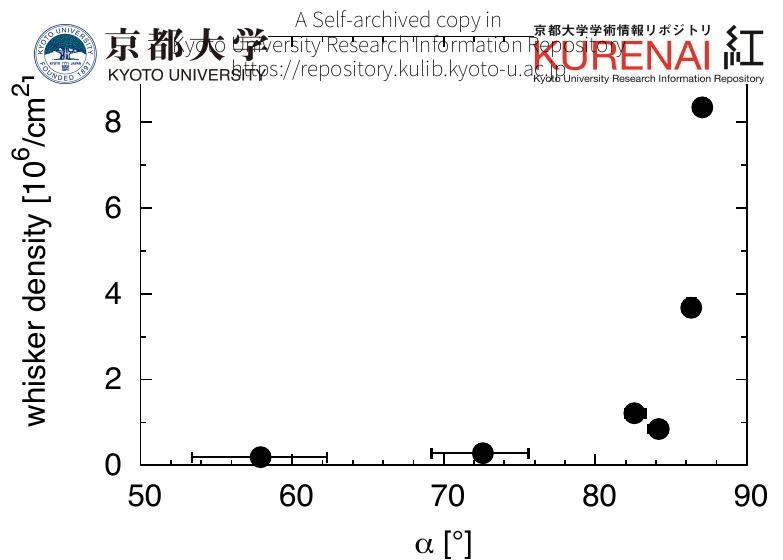




\section{A Self-archived copy in}

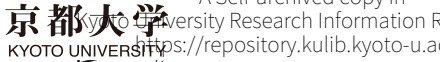
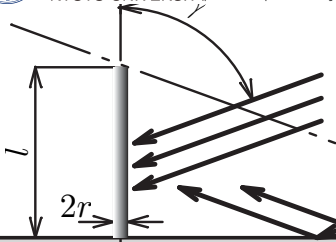

$\cos \gamma$ 


\section{A Self-archived copy in}

京都

京都大学学術情報リポジトリ ב I

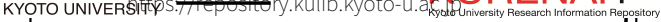

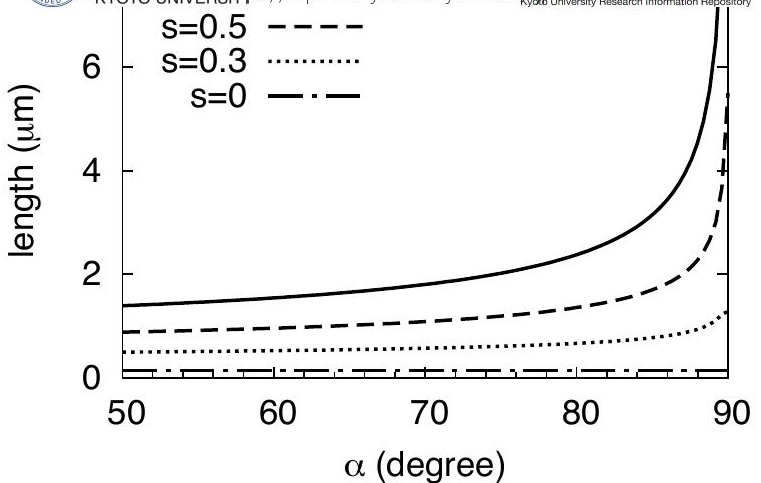

